OPEN ACCESS

Edited by:

Ke-hung Tsui,

Taipei Medical University, Taiwan

Reviewed by:

Riccardo Tellini,

Careggi University Hospital, Italy

Claudia Collà Ruvolo,

University of Naples Federico II, Italy

${ }^{*}$ Correspondence: Kunjie Wang

wangkj@scu.edu.cn

Hong Li

lihonghxhx@scu.edu.cn

${ }^{t}$ These authors have contributed equally to this work and

share first authorship

Specialty section:

This article was submitted to

Genitourinary Oncology,

a section of the journal

Frontiers in Oncology

Received: 11 August 2021 Accepted: 04 October 2021 Published: 18 October 2021

Citation:

Gao X, Zhou L, Ai J, Wang W, Di X,

Peng L, Liao B, Jin $X$, Li H and Wang $K$

(2021) The Impact of Diabetes

on the Prognosis of Upper Tract

Urothelial Carcinoma After Radical

Nephroureterectomy: A Systematic

Review and Meta-Analysis.

Front. Oncol. 11:741145.

doi: 10.3389/fonc.2021.741145

\section{The Impact of Diabetes on the} Prognosis of Upper Tract Urothelial Carcinoma After Radical

\section{Nephroureterectomy: A Systematic Review and Meta-Analysis}

\author{
Xiaoshuai Gao ${ }^{\dagger}$, Liang Zhou ${ }^{\dagger}$, Jianzhong Ai, Wei Wang, Xingpeng Di, Liao Peng, \\ Banghua Liao, Xi Jin, Hong Li ${ }^{*}$ and Kunjie Wang*
}

Department of Urology, Institute of Urology (Laboratory of Reconstructive Urology), West China Hospital, Sichuan University, Chengdu, China

Background: Studies have reported that diabetes is related to the prognosis of upper tract urothelial carcinoma (UTUC) after radical nephroureterectomy (RNU), but this conclusion is still controversial. Here, we performed a meta-analysis to comprehensively explore the association between diabetes and UTUC prognosis.

Methods: In November 2020, we searched PubMed, Web of science and the Cochrane Library to find relevant studies that evaluated the effect of diabetes on the prognosis of UTUC. The Newcastle Ottawa Scale was used to assess the quality of the literature. Review Manager 5.3 was used to pool cancer-specific survival (CSS), overall survival (OS), recurrence-free survival (RFS) and intravesical recurrence (IVR).

Results: A total of 10 studies with 11,303 patients were included in this meta-analysis. Our pooled results showed that diabetes did not affect the survival outcome of UTUC, including CSS (HR: 1.33, 95\% Cl: 0.89-1.98; $P=0.16)$, OS (HR: 1.18, 95\% Cl: 0.77-1.80; $P=0.45$ ) and RFS (HR: 1.37, 95\% Cl: 0.91-2.05; $P=0.13)$. However, diabetes increased the risk of IVR of UTUC patients (HR: 1.26, 95\% Cl: 1.11-1.43; $P=0.0004$ ).

Conclusion: Although diabetes has no significant impact on the survival outcomes of UTUC after RNU, it increases the risk of IVR. Therefore, special attention should be paid to monitoring the IVR for UTUC patients with diabetes and the necessity of appropriate intravesical adjuvant treatment when needed.

Keywords: upper tract urothelial carcinoma, diabetes, radical nephroureterectomy, prognosis, meta-analysis 


\section{INTRODUCTION}

Upper tract urothelial carcinoma (UTUC) is a rare cancer with a yearly incidence of only 1 to 2 cases per 100,000 individuals (1). Although the incidence of UTUC is low, it is always malignant and locally invasive (2). Therefore, radical nephroureterectomy (RNU) is the current gold standard for UTUC treatment (3). Even after RNU, the risk of UTUC recurrence and related mortality is still high. The five-year overall mortality rate in UTUC patients treated with RNU is approximately $40 \%$, and the cancer-specific mortality rate is as high as $25 \%(3,4)$. Therefore, it is necessary to study the prognostic factors of UTUC.

Current prognostic models are based on preoperative factors like tumor multifocality, size, location, tumor stage and grade on biopsy, hydronephrosis $(5,6)$ and postoperative predictors such as T stage, lymphovascular invasion, tumor necrosis, architecture and concomitant carcinoma in situ $(5,7)$. These prognostic factors classified UTUC as low risk or high risk and determined whether the patients should undergo kidneysparing procedures or RNU. Additionally, these models are very important for predicting the survival outcomes of UTUC patients and whether they will be treated with adjuvant chemotherapy after surgery (8). However, these predictive models have limited their clinical applications due to low accuracy. Therefore, it is necessary to find new predictive factors for UTUC patients to increase the accuracy of prognostic models.

In recent years, the incidence of diabetes has increased with changes in diet and lifestyle. Moreover, there are studies showing that diabetes increases the risk of cancer of the liver, colorectum, breast, endometrium and pancreas (9). In addition, diabetes is also an important risk factor for bladder cancer and prostate cancer $(10,11)$. To date, several studies have also shown that diabetes is related to UTUC prognosis, but this conclusion is still controversial. We aimed to explore the effects of diabetes on the prognosis of UTUC through this meta-analysis and draw conclusions with a stronger evidence to guide clinical practice of UTUC treatment.

\section{MATERIALS AND METHODS}

\section{Search Strategy}

Two researchers searched PubMed, Web of science and the Cochrane Library in November 2020 according the PRISMA guidelines (12). They searched for studies on diabetes and the prognosis of UTUC using the following key terms: ("diabetes" OR "glycemic") AND ("upper tract urothelial carcinoma" OR "upper tract urinary carcinoma") AND ("survival" OR "prognostic" OR “progression” OR "recurrence”).

\section{Inclusion and Exclusion Criteria}

We developed the inclusion and exclusion criteria according to the populations, interventions, comparators, outcomes, and study designs (PICOS). Studies were considered eligible if they met the following selection criteria: UTUC patients (populations) after RNU (interventions), studies evaluating the association of diabetes (comparators) with UTUC prognosis (outcomes). All prospective or retrospective controlled trials (study designs) were included in this meta-analysis. The exclusion criteria included the following items: reviews, case series reports, abstract and letters. The study did not include any indicator and its corresponding HR and 95\% intervals of cancerspecific survival (CSS), recurrence-free survival (RFS), overall survival (OS) or intravesical recurrence (IVR). In cases of repeated publications, only the largest publication was included.

\section{Data Extraction and Quality Assessment}

Two researchers (GXS and WW) independently extracted data from the included articles. Any disagreements were resolved by consulting a third researcher (DXP). The following variables were extracted: first author, publication time, study period, patient number, patient age and sex ratio, follow-up time, number of diabetes cases, recurrence, mortality, cancer-specific mortality, adjuvant chemotherapy, and HR and 95\% intervals of CSS, RFS, IVR and OS. The Newcastle Ottawa Scale (NOS) was used to evaluate the quality of the selected studies (13).

\section{Statistical Analysis}

For CSS, RFS, IVR and OS, we pooled the HRs with 95\% CIs to evaluate the effect of diabetes on UTUC. Q test and $\mathrm{I}^{2}$ test were used to evaluated the heterogeneity among studies. The random effects model was performed for high heterogeneity among the outcomes by $\mathrm{P}<0.05$ or $\mathrm{I}^{2}>50 \%$. Otherwise, the fixed effects model was used. We evaluated publication bias by funnel plots. Sensitivity analysis was evaluated by removing a single study to assess the stability of the meta-analysis. All statistical analyses were performed using Review Manager software version 5.3.

\section{RESULTS}

\section{Literature Screening and Quality Assessment}

We initially identified 672 studies from the three databases mentioned above. Ten studies were ultimately included in this meta-analysis based on our inclusion and exclusion criteria (1423). A total of 12,865 patients had UTUC, and 2045 (15.9\%) had diabetes. The literature screening process is shown in Figure 1. The clinical characteristics of the included studies are listed in Table 1. The median age of patients in all included studies was over 60 years old. All included studies were retrospective controlled trials, with NOS scores ranging from 7 to 8 . The details scores are listed in Table $\mathbf{1 .}$

\section{Survival Outcomes}

Six eligible studies evaluated the impact of diabetes on CSS in UTUC $(14,16,17,19,21,23)$. As the heterogeneity among studies was high, a random effects model was used $\left(P=0.0004, I^{2}=78 \%\right)$. Pooled results showed that there was no relationship between diabetes and CSS (HR: 1.33, 95\% CI: 0.89-1.98; $P=0.16$; Figure 2). Four eligible studies $(14,15,19,23)$ evaluated the impact of 


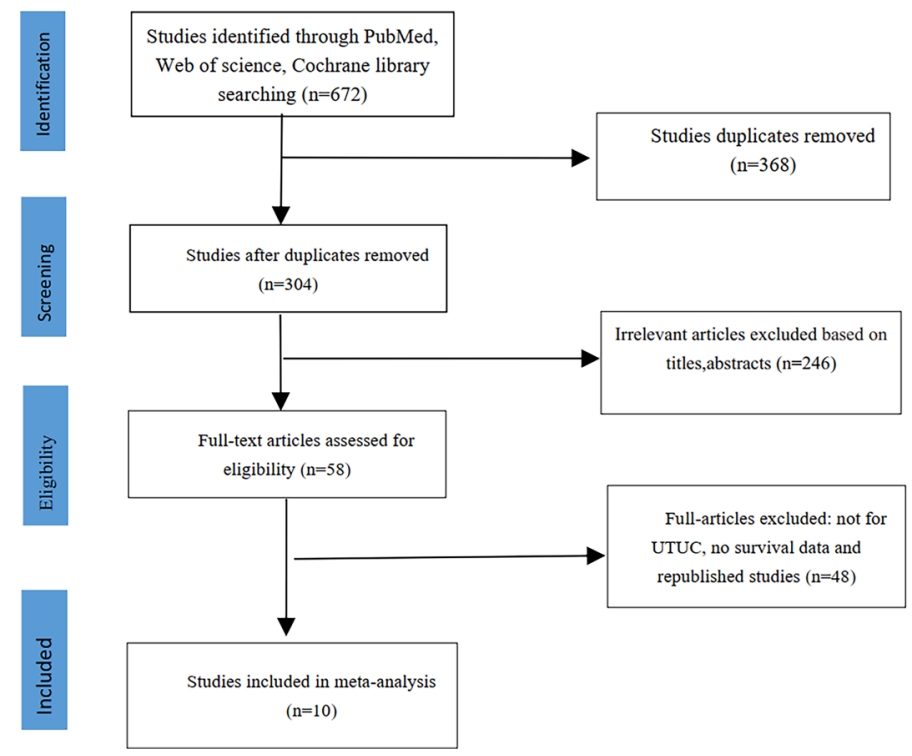

FIGURE 1 | The flowchart showing study search and selection process.

diabetes on RFS, and the meta-analysis revealed that diabetes did not affect RFS (HR: 1.37, 95\% CI: 0.91-2.05; $P=0.13$; Figure 3). Six studies provided data regarding the OS between the two groups $(14,15,17,19,21,23)$. As high heterogeneity existed among the studies $\left(P=0.0003, I^{2}=79 \%\right)$, we used the random effect model, which showed that the OS was similar between the two groups (HR: 1.18, 95\% CI: 0.77-1.80; $P=0.45$; Figure 4).

\section{Intravesical Recurrence}

Four studies provided IVR data $(16,18,20,22)$. The heterogeneity was low $\left(P=0.22, I^{2}=32 \%\right)$, and a fixed-effects model showed that diabetes significantly increased the risk of IVR in UTUC (HR: 1.26, 95\% CI: 1.11-1.43; $P=0.0004$; Figure 5).

\section{Sensitivity Analysis and Publication Bias}

A funnel plot was used to evaluate publication bias, and no evidence for publication bias was found (Supplementary Figure 1). Sensitivity analysis was performed by removing a single study to assess the stability of the meta-analysis. After removing a single study, the pooled $\mathrm{HR}$ and heterogeneity did not change significantly, and no source of heterogeneity was found.

\section{DISCUSSION}

Approximately one-third of UTUC patients will experience early recurrence after RNU, and nearly $80 \%$ of these patients will eventually die due to cancer (24). The current prognostic models are helpful to the selection of surgical methods and the prediction of survival outcomes of UTUC patients, but the accuracy is insufficient (8). Several studies have reported that diabetes may be one of the potential predictors of postoperative survival outcomes in UTUC patients $(14,16,17,19,21,23)$. Therefore, we firstly performed a meta-analysis to evaluate the impact of diabetes on the prognosis outcomes in UTUC patients after RNU. We found that diabetes did not affect any postoperative survival outcomes in UTUC patients. However, it increased the risk of IVR in UTUC. This result implies the importance of paying extra attention to IVR of UTUC patients after RNU and the necessity of appropriate intravesical adjuvant treatment when needed. Moreover, it is recommended that diabetic patients should well-controlled glycemia of UTUC after RNU.

The biological mechanism of IVR in RNU patients is not well understood. At present, there are two widely accepted theories of bladder tumor recurrence: panurothelial field defects and a single transformed cell after descendant intraluminal seeding (25). European Association of Urology guidelines suggest that early intravesical instillation of pirarubicin or mitomycin C after RNU for UTUC could reduce the risk of IVR (26). Therefore, it is particularly important to identify the risk factors for IVR and to intervene in UTUC patients. High-quality research shows that tumor-specific indicators such as multifocality, necrosis, ureteral location, positive preoperative urinary cytology, invasive $\mathrm{pT}$ stage and patient-specific predictors such as previous bladder cancer, male sex, and preoperative chronic kidney disease increase the risk of IVR in UTUC patients (25). A recent multicenter study showed that risk factors such as transurethral resection of the bladder cuff, ureteroscopic biopsy, and positive surgical margins increase IVR for UTUC after RNU (27). We found that diabetes increased the risk of IVR, which is a good supplement to the risk factors for IVR in UTUC patients.

Four studies in our systematic review compared the effect of diabetes on IVR in UTUC patients after RNU $(16,18,20,22)$. 


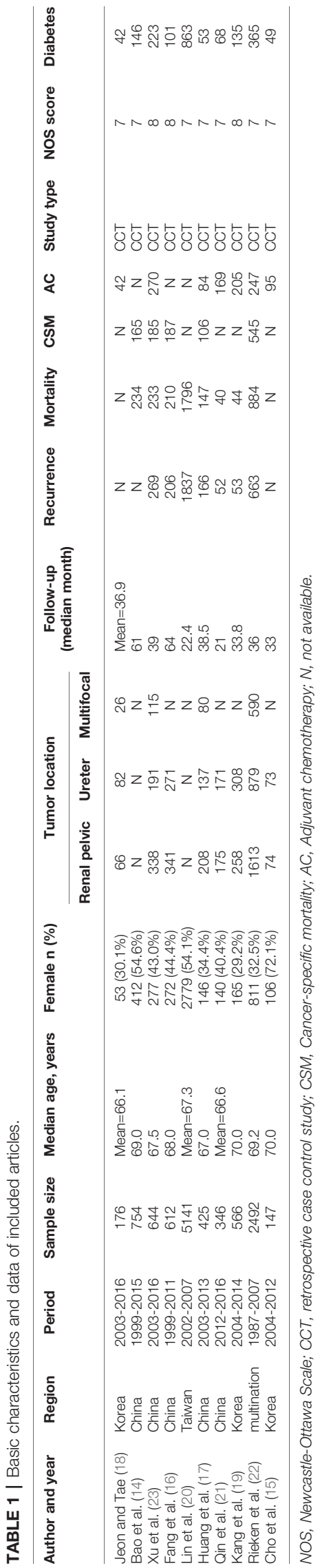

Jeon et al. (18) and Lin et al. (20) found that diabetes increases IVR in UTUC patients. Our meta-analysis supports the conclusions of Jeon et al. (18) and Lin et al. (20) showing that diabetes significantly increases IVR in UTUC patients. However, Fang et al. (16) and Rieken et al. (22) showed that the effect of diabetes on IVR is unclear. Diabetes medication may be one of the reasons for this difference. Diabetes that is not treated with metformin results in an increase in disease recurrence in UTUC patients after RNU (HR, 1.43; $\mathrm{P}=0.01)$, and this effect disappears after taking metformin (HR, 0.90; $\mathrm{P}=0.49)$ (22). In addition, the difference in tumor indicators between studies is an important factor that cannot be ignored regarding IVR in UTUC patients.

The mechanisms underlying the association of diabetes with IVR in UTUC patients are unclear. Hyperglycemia not only provides more nutrients for tumor cells but also activates insulin or insulin-like growth factor 1 (28). Insulin-like growth factor 1 promotes tumor cell proliferation and inhibits apoptosis (29). In addition, hyperglycemia affects many biological functions of cancer cells, such as proliferation, invasion, migration, and recurrence (30). The effect of hyperglycemia on urothelial cell tumors has also been extensively studied. Overexpression of insulin-like growth factor 1 in bladder urothelial cancer cells can promote proliferation and inhibit apoptosis (31). In addition, the immunity of diabetic patients is relatively low, and urinary tract inflammation will also affect the prognosis of UTUC.

Our meta-analysis included 10 retrospective studies comparing the effect of diabetes on the survival outcomes of UTUC. The results are different in these studies. Kang et al. (19) and Huang et al. (17) found that diabetes is associated with worse CSS, RFS and OS. However, Cho et al. (15), Xu et al. (23), Bao et al. (14), Qin et al. (21) and Fang et al. (16) demonstrated that there was no statistically significant difference in the effect of diabetes on UTUC survival outcomes. Our pooled results also showed that diabetes was not associated with worse survival outcomes. The different results among studies can be explained by the following factors.

Recent evidence reveals that many patients and tumor-related factors affect the prognosis of UTUC after RNU. Suzuki et al. (32) and $\mathrm{Xu}$ et al. (33) revealed that higher controlling nutritional status (CONUT) score, greater age, lower BMI, higher CRP, higher $\mathrm{pT}$ stage, higher tumor grade, higher tumor size, concomitant variant histology $(\mathrm{CVH})$, no curative treatment and no usage of pembrolizumab after the diagnosis of UTUC are independent and significant adverse prognostic factors. Besides, Xu et al. (33) demonstrated that CONUT score was an independent predictor for CSS, RFS and OS. Moreover, Marcq et al. (34) performed an international collaborative study to evaluate the predictive value of a new classification of UTUC for kidney-sparing surgery. They found that the higher age, biopsy, high-grade cytology, sessile tumor, hydronephrosis and non-organ-confined disease on preoperative imaging were independently associated with muscle invasion at RNU (34).

Glycemic control for diabetes patients may affect the survival indicators of UTUC. Kang et al. (19) found that poorly controlled diabetes (no DM vs HbAlc > 7) was associated with worse CSS (HR, 2.96; $\mathrm{P}=0.001$ ), RFS (HR, 2.26; $\mathrm{P}=0.003$ ) and $\mathrm{OS}$ (HR, 2.13; $\mathrm{P}=0.001)$. However, there was no significant difference in the effect 


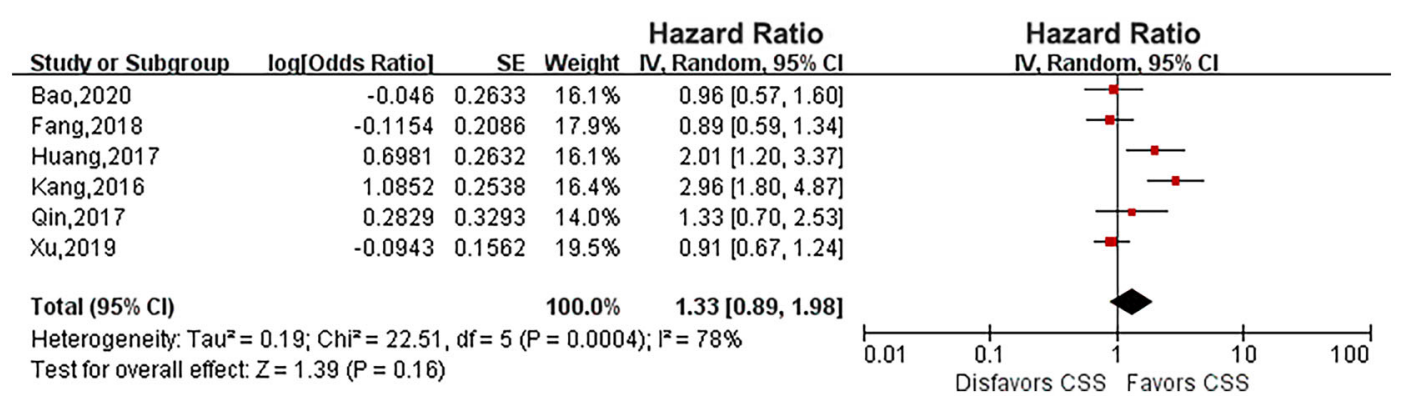

FIGURE 2 | Forest plot of the impact of diabetes on cancer-specific survival.

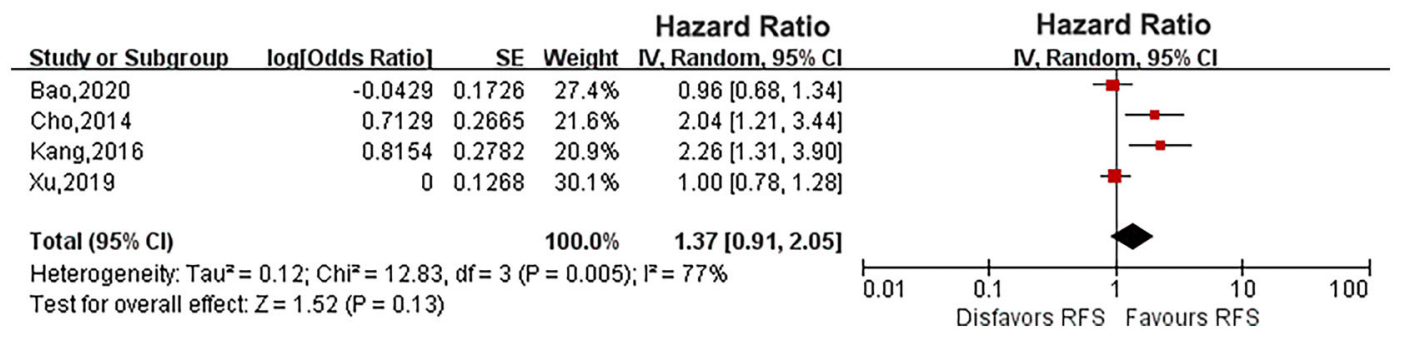

FIGURE 3 | Forest plot of the impact of diabetes on recurrence-free survival.



FIGURE 4 | Forest plot of the impact of diabetes on overall survival.

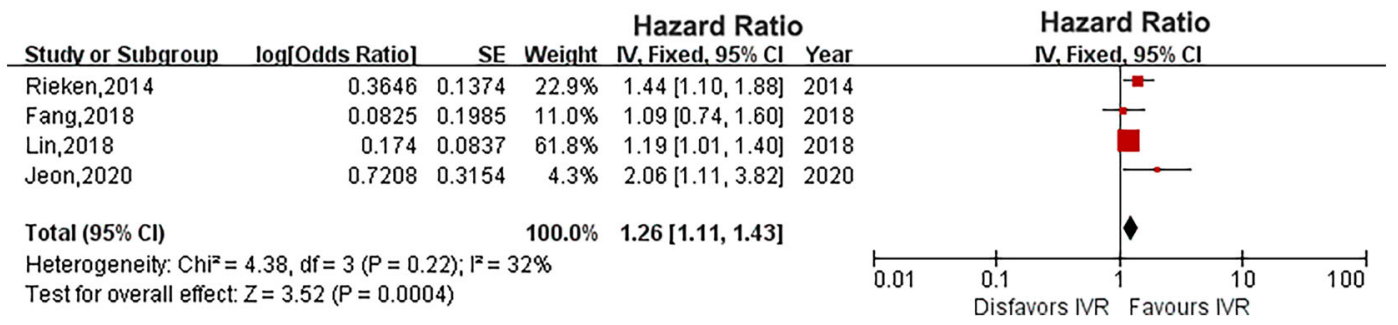

FIGURE 5 | Forest plot of the impact of diabetes on intravesical recurrence. 
of diabetes on UTUC survival indicators in patients with wellcontrolled glycemia (no DM vs HbA1c < 7). Rieken et al. (22) also found that diabetic patients who did not treated with metformin were at significantly higher risk of disease recurrence and cancerspecific death compared to nondiabetic patients and diabetic patients who used metformin. Moreover, previous meta-analysis indicated that metformin intake was associated with an improved RFS ( $\mathrm{HR}=0.55 ; \mathrm{P}=0.01$ ), increased progression-free survival $(\mathrm{HR}=0.70 ; \mathrm{P}=0.03)$, and prolonged CSS $(\mathrm{HR}=0.57 ; \mathrm{P}=0.002)$ (35). In fact, smoking can also interfere with the prognosis of UTUC (36). A meta-analysis shows that smoking increases recurrence and death of UTUC after RNU. Besides, Tellini et al. (37) found that smoking increased risk for major postoperative complications, infections, and mortality of patients treated with radical cystectomy for urothelial bladder cancer.

Our meta-analysis inevitably has some limitations. First, the 10 studies we included were all retrospective studies, and there may be some selection bias. Second, there were insufficient data to pool for medication use in diabetic patients (such as drug types and blood glucose levels after taking the drug). Third, the follow-up time of most studies is not long enough, which may be the reason why many differences in survival outcomes are not reflected, so it also suggests the need for longer follow-up studies. Fourth, limitations of the data in the included studies prevented analysis of interference from some factors that may affect the prognosis of UTUC, such as patient age, sex, smoking status, and drinking habits. Nevertheless, we found that diabetes can increase IVR of UTUC patients after RNU. We hope that the emergence of large-scale prospective studies in the future can further support our conclusions.

\section{CONCLUSIONS}

Although diabetes has no significant impact on the survival outcome of UTUC after RNU, it increases the risk of IVR. Therefore, special attention should be paid to monitoring the IVR for UTUC patients with diabetes and the necessity of appropriate intravesical adjuvant treatment when needed.

\section{REFERENCES}

1. Soria F, Shariat SF, Lerner SP, Fritsche HM, Rink M, Kassouf W, et al. Epidemiology, Diagnosis, Preoperative Evaluation and Prognostic Assessment of Upper-Tract Urothelial Carcinoma (UTUC). World J Urol (2017) 35(3):379-87. doi: 10.1007/s00345-016-1928-x

2. Chromecki TF, Cha EK, Fajkovic H, Margulis V, Novara G, Scherr DS, et al. The Impact of Tumor Multifocality on Outcomes in Patients Treated With Radical Nephroureterectomy. Eur Urol (2012) 61(2):245-53. doi: 10.1016/ j.eururo.2011.09.017

3. Ploussard G, Xylinas E, Lotan Y, Novara G, Margulis V, Rouprêt M, et al. Conditional Survival After Radical Nephroureterectomy for Upper Tract Carcinoma. Eur Urol (2015) 67(4):803-12. doi: 10.1016/j.eururo.2014.08.003

4. Rink M, Xylinas E, Trinh QD, Lotan Y, Margulis V, Raman JD, et al. GenderSpecific Effect of Smoking on Upper Tract Urothelial Carcinoma Outcomes. BJU Int (2013) 112(5):623-37. doi: 10.1111/bju.12014

5. Rouprêt M, Babjuk M, Compérat E, Zigeuner R, Sylvester RJ, Burger M, et al. European Association of Urology Guidelines on Upper Urinary Tract

\section{DATA AVAILABILITY STATEMENT}

The original contributions presented in the study are included in the article/Supplementary Material. Further inquiries can be directed to the corresponding authors.

\section{AUTHOR CONTRIBUTIONS}

XG wrote the manuscript writing. LZ collected and analyzed the data. JA, WW, and XD analyzed the data. LP, XJ, and BL helped review and revise the manuscript. HL and KW helped design the study and revise article. All authors contributed to the article and approved the submitted version.

\section{FUNDING}

This study was supported by the 1.3 .5 project for disciplines of excellence, West China Hospital, Sichuan University (ZYGD18011, ZY2016104 and ZYJC18015); Project of Science and Technology Department of Sichuan Province (2021YFS0116 and 2020YFS0047); Chengdu International Science and Technology Cooperation Funding (2019-GH02-00011-HZ); Project of Guangzhou Medical University (HX-H1701002).

\section{ACKNOWLEDGMENTS}

We are grateful to the authors and patients of the included studies.

\section{SUPPLEMENTARY MATERIAL}

The Supplementary Material for this article can be found online at: https://www.frontiersin.org/articles/10.3389/fonc.2021. 741145/full\#supplementary-material

Supplementary Figure 1 | Funnel plot evaluating the publication bias. Cancerspecific survival (A), recurrence-free survival (B), overall survival (C), intravesical recurrence (D).

Urothelial Carcinoma: 2017 Update. Eur Urol (2018) 73(1):111-22. doi: 10.1016/j.eururo.2017.07.036

6. Kim HS, Jeong CW, Kwak C, Kim HH, Ku JH. Association Between Demographic Factors and Prognosis in Urothelial Carcinoma of the Upper Urinary Tract: A Systematic Review and Meta-Analysis. Oncotarget (2017) 8 (5):7464-76. doi: 10.18632/oncotarget.10708

7. Gao X, Ma Y, Chen G, Chen J, Li H, Li H, et al. Concomitant Carcinoma in Situ as a Prognostic Factor in the Upper Tract Urothelial Carcinoma After Radical Nephroureterectomy: A Systematic Review and Meta-Analysis. Urol Oncol (2020) 38(6):574-81. doi: 10.1016/j.urolonc.2020.02.020

8. Shao Y, Li W, Wang D, Wu B. Prognostic Value of Preoperative LymphocyteRelated Systemic Inflammatory Biomarkers in Upper Tract Urothelial Carcinoma Patients Treated With Radical Nephroureterectomy: A Systematic Review and Meta-Analysis. World J Surg Oncol (2020) 18 (1):273. doi: 10.1186/s12957-020-02048-7

9. Joost HG. Diabetes and Cancer: Epidemiology and Potential Mechanisms. Diabetes Vasc Dis Res (2014) 11(6):390-4. doi: 10.1177/147916411 4550813 
10. Zhang F, Yang Y, Skrip L, Hu D, Wang Y, Wong C, et al. Diabetes Mellitus and Risk of Prostate Cancer: An Updated Meta-Analysis Based on 12 CaseControl and 25 Cohort Studies. Acta Diabetol (2012) 49 Suppl 1:S235-46. doi: 10.1007/s00592-012-0439-5

11. Xu Y, Huo R, Chen X, Yu X. Diabetes Mellitus and the Risk of Bladder Cancer: A PRISMA-Compliant Meta-Analysis of Cohort Studies. Medicine (Baltimore) (2017) 96(46):e8588. doi: 10.1097/md.0000000000008588

12. Moher D, Liberati A, Tetzlaff J, Altman DG. Preferred Reporting Items for Systematic Reviews and Meta-Analyses: The PRISMA Statement. J Clin Epidemiol (2009) 62(10):1006-12. doi: 10.1016/j.jclinepi.2009.06.005

13. Wells G, Shea B, O'Connell D, Peterson J, Welch V, Losos M, et al. The Newcasstle-Ottawa Scale (NOS) for Assessing the Quality of Non-Randomised Studies in Meta-Analysis. Available at: http://www.ohri.ca/programs/clinical_ epidemiology/oxford.asp (Accessed: September 4, 2020).

14. Bao Z, Li Y, Guan B, Xiong G. High Preoperative Controlling Nutritional Status Score Predicts a Poor Prognosis in Patients With Localized Upper Tract Urothelial Cancer: A Propensity Score Matching Study in a Large Chinese Center. Cancer Manag Res (2020) 12:323-35. doi: 10.2147/cmar.s225711

15. Cho YH, Seo YH, Chung SJ, Hwang I, Yu HS, Kim SO, et al. Predictors of Intravesical Recurrence After Radical Nephroureterectomy for Upper Urinary Tract Urothelial Carcinoma: An Inflammation-Based Prognostic Score. Korean J Urol (2014) 55(7):453-9. doi: 10.4111/kju.2014.55.7.453

16. Fang D, He S, Xiong G, Singla N, Cao Z, Zhang L, et al. Comparison of Clinicopathologic Characteristics, Epigenetic Biomarkers and Prognosis Between Renal Pelvic and Ureteral Tumors in Upper Tract Urothelial Carcinoma. BMC Urol (2018) 18(1):22. doi: 10.1186/s12894-018-0334-7

17. Huang J, Wang Y, Yuan Y, Chen Y, Kong W, Chen H, et al. Preoperative Serum Pre-Albumin as an Independent Prognostic Indicator in Patients With Localized Upper Tract Urothelial Carcinoma After Radical Nephroureterectomy. Oncotarget (2017) 8(22):36772-79. doi: 10.18632/oncotarget.13694

18. Jeon BJ, Tae BS. Preoperative Sterile Pyuria as a Prognostic Biomarker for Intravesical Recurrence in Upper Urinary Tract Urothelial Carcinoma. Investig Clin Urol (2020) 61: (1):51-8. doi: 10.4111/icu.2020.61.1.51

19. Kang SG, Hwang EC, Jung SI, Yu HS, Chung HS, Kang TW, et al. Poor Preoperative Glycemic Control Is Associated With Dismal Prognosis After Radical Nephroureterectomy for Upper Tract Urothelial Carcinoma: A Korean Multicenter Study. Cancer Res Treat (2016) 48(4):1293-301. doi: $10.4143 /$ crt.2016.021

20. Lin MY, Li WM, Huang CN, Lee HL, Niu SW, Chen LT, et al. Dialysis Increases the Risk of Bladder Recurrence in Patients With Upper Tract Urothelial Cancer: A Population-Based Study. Ann Surg Oncol (2018) 25 (4):1086-93. doi: 10.1245/s10434-017-6295-3

21. Qin C, Liang EL, Du ZY, Qiu XY, Tang G, Chen FR, et al. Prognostic Significance of Urothelial Carcinoma With Divergent Differentiation in Upper Urinary Tract After Radical Nephroureterectomy Without Metastatic Diseases: A Retrospective Cohort Study. Medicine (Baltimore) (2017) 96(21): e6945. doi: 10.1097/md.0000000000006945

22. Rieken M, Xylinas E, Kluth L, Trinh QD, Lee RK, Fajkovic H, et al. Diabetes Mellitus Without Metformin Intake is Associated With Worse Oncologic Outcomes After Radical Nephroureterectomy for Upper Tract Urothelial Carcinoma. Eur J Surg Oncol (2014) 40(1):113-20. doi: 10.1016/j.ejso. 2013.09.016

23. Xu H, Tan P, Zheng X, Ai J, Lin T, Jin X, et al. Metabolic Syndrome and Upper Tract Urothelial Carcinoma: A Retrospective Analysis From a Large Chinese Cohort. Urol Oncol (2019) 37(4):291.e19-91.e28. doi: 10.1016/j.urolonc. 2018.12.005

24. Cha EK, Shariat SF, Kormaksson M, Novara G, Chromecki TF, Scherr DS, et al. Predicting Clinical Outcomes After Radical Nephroureterectomy for Upper Tract Urothelial Carcinoma. Eur Urol (2012) 61(4):818-25. doi: 10.1016/j.eururo.2012.01.021

25. Seisen T, Granger B, Colin P, Léon P, Utard G, Renard-Penna R, et al. A Systematic Review and Meta-Analysis of Clinicopathologic Factors Linked to Intravesical Recurrence After Radical Nephroureterectomy to Treat Upper Tract Urothelial Carcinoma. Eur Urol (2015) 67(6):1122-33. doi: 10.1016/ j.eururo.2014.11.035
26. Rouprêt M, Babjuk M, Compérat E, Zigeuner R, Sylvester R, Burger M, et al. European Guidelines on Upper Tract Urothelial Carcinomas: 2013 Update. Eur Urol (2013) 63(6):1059-71. doi: 10.1016/j.eururo.2013.03.032

27. Katims AB, Say R, Derweesh I, Uzzo R, Minervini A, Wu Z, et al. Risk Factors for Intravesical Recurrence After Minimally Invasive Nephroureterectomy for Upper Tract Urothelial Cancer (ROBUUST Collaboration). J Urol (2021) 206 (3):568-76. doi: 10.1097/ju.0000000000001786

28. Suikkari AM, Koivisto VA, Rutanen EM, Yki-Järvinen H, Karonen SL, Seppälä M. Insulin Regulates the Serum Levels of Low Molecular Weight Insulin-Like Growth Factor-Binding Protein. J Clin Endocrinol Metab (1988) 66(2):266-72. doi: 10.1210/jcem-66-2-266

29. Iwamura M, Ishibe M, Sluss PM, Cockett AT. Characterization of Insulin-Like Growth Factor I Binding Sites in Human Bladder Cancer Cell Lines. Urol Res (1993) 21(1):27-32. doi: 10.1007/bf00295188

30. Duan W, Shen X, Lei J, Xu Q, Yu Y, Li R. Hyperglycemia, a Neglected Factor During Cancer Progression. Biomed Res Int (2014) 2014:461917. doi: 10.1155/ 2014/461917

31. Metalli D, Lovat F, Tripodi F, Genua M, Xu SQ, Spinelli M, et al. The InsulinLike Growth Factor Receptor I Promotes Motility and Invasion of Bladder Cancer Cells Through Akt- and Mitogen-Activated Protein KinaseDependent Activation of Paxillin. Am J Pathol (2010) 176(6):2997-3006. doi: 10.2353/ajpath.2010.090904

32. Suzuki H, Ito M, Takemura K, Nakanishi Y, Kataoka M, Sakamoto K, et al. Prognostic Significance of the Controlling Nutritional Status (CONUT) Score in Advanced Urothelial Carcinoma Patients. Urol Oncol (2020) 38(3):76.e117. doi: 10.1016/j.urolonc.2019.10.014

33. $\mathrm{Xu} \mathrm{H}$, Tan $\mathrm{P}$, Jin $\mathrm{X}$, Ai J, Lin T, Lei H, et al. Validation of the Preoperative Controlling Nutritional Status Score as an Independent Predictor in a Large Chinese Cohort of Patients With Upper Tract Urothelial Carcinoma. Cancer Med (2018) 7(12):6112-23. doi: 10.1002/cam4.1902

34. Marcq G, Foerster B, Abufaraj M, Matin SF, Azizi M, Gupta M, et al. Novel Classification for Upper Tract Urothelial Carcinoma to Better Risk-Stratify Patients Eligible for Kidney-Sparing Strategies: An International Collaborative Study. Eur Urol Focus (2021). doi: 10.1016/j.euf.2021.03.018

35. Hu J, Chen JB, Cui Y, Zhu YW, Ren WB, Zhou X, et al. Association of Metformin Intake With Bladder Cancer Risk and Oncologic Outcomes in Type 2 Diabetes Mellitus Patients: A Systematic Review and Meta-Analysis. Medicine (Baltimore) (2018) 97(30):e11596. doi: 10.1097/md.0000000000011596

36. van Osch FH, Jochems SH, van Schooten FJ, Bryan RT, Zeegers MP. Significant Role of Lifetime Cigarette Smoking in Worsening Bladder Cancer and Upper Tract Urothelial Carcinoma Prognosis: A Meta-Analysis. J Urol (2016) 195(4 Pt 1):872-9. doi: 10.1016/j.juro.2015.10.139

37. Tellini R, Mari A, Muto G, Cacciamani GE, Ferro M, Stangl-Kremser J, et al. Impact of Smoking Habit on Perioperative Morbidity in Patients Treated With Radical Cystectomy for Urothelial Bladder Cancer: A Systematic Review and Meta-Analysis. Eur Urol Oncol (2021) 4(4):580-93. doi: 10.1016/ j.euo.2020.10.006

Conflict of Interest: The authors declare that the research was conducted in the absence of any commercial or financial relationships that could be construed as a potential conflict of interest.

Publisher's Note: All claims expressed in this article are solely those of the authors and do not necessarily represent those of their affiliated organizations, or those of the publisher, the editors and the reviewers. Any product that may be evaluated in this article, or claim that may be made by its manufacturer, is not guaranteed or endorsed by the publisher.

Copyright (c) 2021 Gao, Zhou, Ai, Wang, Di, Peng, Liao, Jin, Li and Wang. This is an open-access article distributed under the terms of the Creative Commons Attribution License (CC BY). The use, distribution or reproduction in other forums is permitted, provided the original author(s) and the copyright owner(s) are credited and that the original publication in this journal is cited, in accordance with accepted academic practice. No use, distribution or reproduction is permitted which does not comply with these terms. 